Safety 2 is an emerging concept, advocating for learning from "what goes right", rather than "what goes wrong”. Currently, Safety 2 methodology is not well described in healthcare.

Intervention We piloted a system of peer-reporting for episodes of clinical excellence, to identify common themes of excellence practice. The "Learning from excellence" initiative was launched in April 2014 in our PICU. Reporting was accessible to all staff via a simple online form. Excellence was identified by individual reporters: a standardised definition of excellence was not provided.

Study design This is a descriptive study of themes identified from a system of peer-reported excellence in healthcare. All reports were analysed for themes in 2 domains:

1. Excellent practice (i.e. what was done?)

2. Clinical context.

Strategy for change The project was designed in conjunction with the Trust governance department. An online reporting form was published on the hospital intranet, and the initiative was championed by members of the PICU team. Reported individuals and teams were notified of their excellence citation via email. Briefings with summaries of excellence reports, highlighting learning opportunities are circulated to the department weekly. The next phase is to include the contents of these reports in the educational programme to develop role modelling and emulation of excellent practice. This process is underway at the time of writing.

Measurement of improvement To date, 74 reports have been submitted and analysed. Staff members from every clinical group have contributed to the reporting, and received reports. 19 practice themes and 24 discrete clinical context themes have been identified. Table 1 shows the 10 commonest themes in each domain.

Effects of changes There is a general trend of increasing numbers of reports since launch. The scope of the project is increasing through reporting outside the PICU. This has occurred without external championing, presumably via a "viral" spread.

At the time of writing we have not evaluated staff satisfaction or any impact on clinical metrics. However, the rising frequency of reporting, and the spread outside the unit provides evidence of proof of concept, and acceptance of potential utility.

Lessons learnt Involvement of the Trust governance department allowed us to use an existing infrastructure for reporting, and provided useful insights into safety management.

Championing within the environment of the intervention was essential.

\begin{tabular}{|c|c|c|c|}
\hline Practice theme & Occurrences & Clinical context theme & Occurrences \\
\hline Peer support & 17 & Prescribing & 7 \\
\hline Preparedness/planning & 12 & End of life & 6 \\
\hline Compassionate care & 5 & Admissions & 5 \\
\hline Timeliness & 5 & Resuscitation & 5 \\
\hline Clinical leadership & 4 & Sepsis & 5 \\
\hline Advocacy/courage & 4 & Complex discharges & 4 \\
\hline Communication with & & Arterial line & \\
\hline colleagues & 3 & management & 3 \\
\hline Communication with & & Expressed Breast & \\
\hline patients & 3 & Milk delivery & 2 \\
\hline Going the extra mile & 3 & Handover & 2 \\
\hline Teamwork & 3 & Transfer & 2 \\
\hline
\end{tabular}

Message for others We have demonstrated that peer-reported excellence can be captured from a wide range of themes and clinical contexts. We intend to use these reports to generate a positive movement to change, to inspire excellent practice through role modelling and emulation.

\section{G550(P) ASSESSING COMMUNICATION IN PHLEBOTOMY SERVICES IN AN IN-PATIENT SETTING}

B Singh, S Habeeb, J Pitchforth, C Lemer. General Paediatrics, Evalina Children's Hospital, London, UK

\subsection{6/archdischild-2015-308599.500}

Context Quality improvement project carried out on the general paediatrics ward involving the different medical teams, (general paediatrics, surgery, ENT, orthopaedics), the Nursing team and the phlebotomists

Problem We addressed the problem of the delay in the notification of failed blood tests from the phlebotomy team to the relevant medical team. This was identified to affect patient care by causing a delay in diagnosis, decisions about treatment and discharge, leading on to prolonging patient stay, this in affects the overall patient experience.

Assessment of problem and analysis of its causes The problem was quantified by assessing the total number of unsuccessful blood tests in 1 week and finding out how many of those had been communicated to the relevant medical team at the time. To understand how there could be possible breakdowns in communication, a day was spent with the phlebotomists to understand how they prioritise their jobs, how long they have per patient and what would be the best way for them to contact the medical team. The staff involved included junior doctors, phlebotomists and nurses.

Intervention At the end of each phlebotomy round, the phlebotomists bleep the nurse in charge for the ward and inform of any unsuccessful blood tests. The nurse in charge can then contact the relevant medical team so that they can act and decide whether the test is needed more urgently or can wait until the next phlebotomy round.

Strategy for change The change was implemented by being discussed at the weekly grand round session so that all staff were aware. Staff involved in the change included the medical team, nursing team and phlebotomists. The results were presented at an audit meeting and a re-audit was performed 1 year later showing a $100 \%$ success rate and that change had been sustained. These were results were then represented at junior

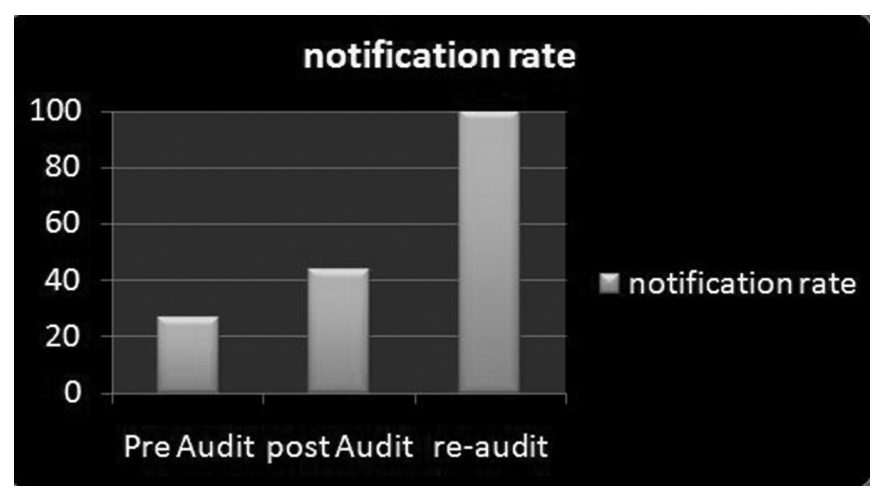

Abstract G550(P) Figure 1 
doctor teaching and discussed in clinical governance breakfast meeting.

Measurement of improvement Pre intervention audit, 30 blood tests requested in 1 week, 11 unsuccessful, pre-intervention notification $27 \%$ There was a re-audit 2 weeks after intervention which showed 46 total blood requests in 1 week, 21 unsuccessful, post intervention notification 44\% showing an improvement. A further re-audit done 1 year later showed 25 total blood tests requested in 1 week, 6 unsuccessful, 100\% notification which confirmed sustained change in practice. The same methods were used in data collection as had been in the original data collection.

Effects of changes This change created a more effective way for phlebotomists to communicate with the relevant medical team through the nurse in charge which would not compromise their time to do their job. It also allowed the medical team to act in a timely manner in the knowledge of an unsuccessful blood tests to decide how to act. It improved working relations with the doctors and phlebotomists. This change completely resolved the problem which triggered our original work and has allowed us to try to seek further ways in which the service can be more efficient.

Lessons learnt This work has taught me the importance of learning first what process is in place before setting about to make changes. I feel this project was successful because we first looked at what the phlebotomists did in their role. We created process maps to understand how the current process was working. Next time I would consider involving other wards.

Message for others Change can happen and be sustained as has been seen with our re-audit. This has meant that patient care has improved and we have minimised one of the causes of delays to decisions about patient care.

\section{G551(P) INTRODUCING A PAEDIATRIC MENTORSHIP SCHEME FOR TRAINEES IN THE SOUTH WEST REGION}

${ }^{1}$ PT van Hensbergen, ${ }^{2} \mathrm{JC}$ Sconce. 'Paediatric Department, Taunton and Somerset NHS Trust, Taunton, UK; ${ }^{2}$ Paediatric Department, Swindon and Marlborough Hospitals NHS Trust, Swindon, UK

\subsection{6/archdischild-2015-308599.501}

Context We developed a mentorship scheme for new paediatric doctors (Specialty Training Level 1) in our deanery. Mentoring is "a process of helping, supporting and encouraging paediatricians to develop their skills and to maximise their potential performance". 1 The deanery is responsible for training 150 paediatric doctors across seven hospital trusts.

Specific problem Maintaining focus on a paediatric trainee's individual career interests can be challenging in light of higher clinical workload, staffing shortages and increasing requirement for continuous standardised assessments.

Assessment of problem Paediatric services require large-scale transitions in the context of increasing workload pressures coupled to huge economic limitation. ${ }^{2}$ It is essential the workforce approaches these changes in a positive, motivated way.

Mentoring is well-established throughout different industries and organisations. It is a cost-effective, powerful personal development tool that encourages, supports and guides trainees in their individual goals. We anticipate this will benefit all parties:
- Junior trainees can readily access a friendly, reliable and supportive mentor with good working knowledge of the deanery, helpful contacts and experience of taking projects forward

- Senior trainees will improve mentoring, coaching and supervisory skills through a formal training course and develop these through practical application

- Patients and colleagues will benefit from motivated and enthusiastic trainees whose own educational and training needs are being considered

A recent study from the London Paediatric Deanery ${ }^{3}$ highlighted "a high demand for peer mentoring in paediatrics". Their programme was highly valued by participants, who gained "significant benefits including acquisition of transferable skills and positive changes in behaviour".

Intervention We arranged a mentor training day for senior trainees from the region (ST4-ST8). A full-day workshop was organised, and fully funded, by the Southwest Leadership Academy. Twelve trainees wished to provide mentoring and were able to attend the course, many others registered their interest for future workshops. Delivered content included an introduction to the mentor role, approaches to effective mentoring, mentoring models, and contracting a mentor relationship.

An interactive session followed to discuss how the mentoring programme should be developed. We produced a mentor handbook with further information on the mentor role and detail regarding the programme.

We received excellent written feedback from the day (all trainees would recommend the workshop to colleagues).

Programme design Trainees who successfully completed the mentor workshop were asked to write a 250 -word statement about themselves (11/12 completed, one withdrew for maternity leave). We did not provide further guidance on statement structure or content as we wished to promote individuality and personality.

We delivered a presentation at the ST1 induction day to advertise the scheme. We received very positive feedback, all ST1 trainees $(14 / 14)$ wished to participate.

We asked mentees to review all statements and rank them in order of preference. We were able to match all mentees to one of their "top-three" preferred mentors.

We have arranged an evening for all participants to meet together. Following this, mentor-mentee pairs will meet 2-3 monthly. We anticipate that mentor relationships will continue throughout the ST1-3 period.

Evaluation Feedback will be collected quarterly to evaluate the programme and guide future development. We aim to establish a successful rolling programme that offers effective mentoring to all future ST1 trainees starting in the deanery.

Acknowledgements We benefitted from the experiences of Sarah Eisen and Seema Sukhani, who developed a mentor scheme in the London Deanery. We are grateful for their support and guidance.

\section{REFERENCES}

1 Mentoring and clinical supervision for paediatricians. Paediatrician's Handbook, Chapter 4.6, RCPCH, 2013

$2 \mathrm{RCPCH}$. Facing the future $-\mathrm{A}$ review of paediatric services. April 2011

3 Eisen S, Sukhani S, Brightwell A, Stoneham S, Long A. Peer mentoring: evaluation of a novel programme in paediatrics. Arch Dis Child 2014;99:142-146, doi:10.1136/archdischild-2013-304277 